\title{
Molecular dynamics simulation of phase transitions in crystalline lead (II) fluoride
}

\author{
A. Monteil*, S. Chaussedent, D. Guichaoua \\ LUNAM Université, Université d'Angers, Laboratoire de Photonique d'Angers, EA 4464, 2 bd Lavoisier, 49045 Angers Cedex 01, France
}

\section{H I G H L I G H T S}

- We simulate phase transitions of crystalline lead fluoride.

- We characterize the simulated phases.

- The role of applied pressure and temperature is determined.

- We interpret the transition as a lattice reorganization due to the ionic mobility.

\section{A R T I C L E I N F O}

\section{Article history:}

Received 14 September 2013

Received in revised form

6 February 2014

Accepted 7 March 2014

\section{Keywords:}

Computer modeling and simulation

Molecular dynamics

Phase transitions

Crystal structure

\begin{abstract}
A B S T R A C T
Using classical molecular dynamics applied to crystalline $\mathrm{PbF}_{2}$, we have simulated transitions between cubic and orthorhombic phases and reciprocally. These transitions were induced by pressure and temperature treatments. The orthorhombic phase was evidenced by analysis of different distribution functions and XRD spectra drawn from the ions positions in the simulated samples.
\end{abstract}

(c) 2014 Elsevier B.V. All rights reserved.

\section{Introduction}

Lead (II) fluoride can be found under two crystalline phases: $\beta-\mathrm{PbF}_{2}$ with a cubic fluorite structure (space group $\mathrm{Fm} \overline{3} \mathrm{~m}$ ) and $\alpha-\mathrm{PbF}_{2}$ with an orthorhombic cotunnite structure (space group Pnma). Schematic diagram of the crystalline structures of both phases can be seen on Fig. 2 in the paper of S. Hull and D.A. Keen [1].

Both phases are stable at ambient conditions. However, the $\alpha$ form, which is denser than the $\beta$ form, is more stable for high pressure conditions. In counterpart, the $\beta$ form is more stable for high temperature conditions [1].

The interest for lead fluoride comes from its superionic conducting properties - relatively high ionic conductivity at not high temperatures - [2]. The superionic form of $\mathrm{PbF}_{2}$ is the cubic $\beta$ phase. The fast conduction is interpreted as due to the mobility of anion vacancies [2]. This system has been studied by computer simulation: for the ion mobility as well as for the anionic disorder [3].

\footnotetext{
* Corresponding author. Tel.: +33 241735361.

E-mail address: andre.monteil@univ-angers.fr (A. Monteil).
}

Lead fluoride is a suitable system for the simulation of phase transformations. It has been already modeled in the case of devitrification studies by classical molecular dynamics (MD) numerical simulations [4]. As it was discussed in this paper, the interaction potentials from Walker et al. [5] are well appropriated to obtain consistent values for the ionic mobility and for phase transformations.

Crystalline phase transitions are usually simulated for small systems ( $\sim 100$ atoms) using metadynamics techniques [6] based on the constant pressure Parinello-Rahman Molecular Dynamics method with the size of the simulation box as a parameter. The use of periodic replica in MD simulations of large systems is well adapted for disordered materials (glasses and liquids). However for crystals the simulated box must be adapted to the symmetry of the crystal. In order to avoid imposed symmetries the choice of previous authors has been to introduce the lattice parameters as adjustable ones. Another way, the one we follow in this work, is to use a large enough box: the strains due to the shape are weak enough and are supposed to not impose an a priori crystalline structure. 
This work deals with a numerical study of pressure- and temperature-induced phase's changes in $\mathrm{PbF}_{2}$. We present a detailed analysis of the structural and dynamical parameters of the numerical samples to characterize and explain these phase's changes.

\section{Simulation}

Numerical samples containing $6912 \mathrm{PbF}_{2}$ units were simulated using DL_POLY [7]. Initial configurations were $\beta-\mathrm{PbF}_{2}$ structures in a $71.16 \AA$ sized cube (the simulation box) enclosing $12^{3}$ elementary face centered cubic units. Each $\mathrm{Pb}^{2+}$ is surrounded by $8 \mathrm{~F}^{-}$at a distance of $2.566 \AA$.

Before each simulation, the sample is heated at a given temperature ranging from $300 \mathrm{~K}$ to $800 \mathrm{~K}$. This starting temperature is a feature of the heat treatment which is then applied to the sample during the simulation. The simulation is composed of two successive parts: the first one is to keep the system at the initial temperature in increasing gradually the pressure from 0 up to $70 \mathrm{GPa}$; the second part is to rapidly cool the system at $300 \mathrm{~K}$ and to gradually release its pressure. An example of a heat treatment is given on the Fig. 1, where it can be seen that the whole simulation is composed of successive stages of 40 ps duration. Each stage is itself composed of two parts: the first one maintains constant the temperature and the pressure of the system (NPT ensemble for $20 \mathrm{ps}$ ) and the second one maintains constant its energy and volume (NVE ensemble for $20 \mathrm{ps}$ ). Alternating NPT and NVE stages are justified to relax the system before going on with another pressure-step.

A complete heat treatment is achieved after 700 ps, corresponding to a numerical simulation of 700000 time-steps of $1 \mathrm{fs}$. During the simulation, the lead fluoride appears in three successive phases. In the following discussion, the first one will be denoted $\beta$ phase (the initial structure being $\beta-\mathrm{PbF}_{2}$ ). Before having determined their characteristics, the second and the third ones will be denoted A-phase and B-phase, respectively. For instance, on Fig. 1, the $\beta$-phase is encountered from 0 to $140 \mathrm{ps}$, the A-phase occurs from 140 to 560 ps and the B-phase after 560 ps.

Different physical information were recorded at each stage of the simulation: temperature, pressure, and volume of the system, mean square displacements (MSD) of $\mathrm{Pb}$ and $\mathrm{F}$, ions positions from

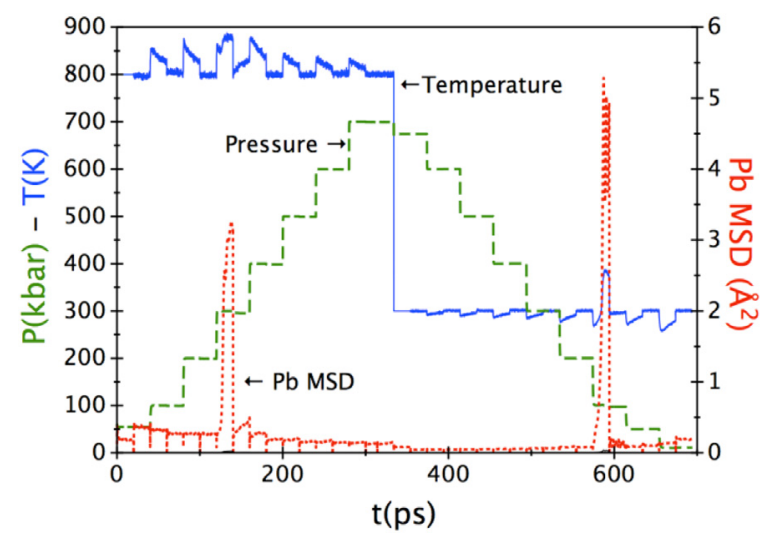

Fig. 1. In these simulations each stage takes 40 ps and is constituted of two parts: at constant temperature and pressure (ensemble NPT during $20 \mathrm{ps}$ ) and constant energy and volume (ensemble NVE during 20 ps). At 340 ps the stage begins with a constant volume and temperature (ensemble NVT during $20 \mathrm{ps}$ ) simulation at $300 \mathrm{~K}$. The first half of the simulation consists to increase gradually the pressure (dash in green) at high temperature (continuous line in blue), the second half to decrease gradually the pressure at room temperature. The dotted red curve, which corresponds to the mean squared displacement of $\mathrm{Pb}^{2+}$ ions, shows two peaks after 140 ps and 580 ps. (For interpretation of the references to color in this figure legend, the reader is referred to the web version of this article.) which snapshots could be drawn as well as radial distribution functions (RDF), cumulative distribution function (CDF), angular distribution functions and X-ray diffraction pattern (XRD) could be calculated. As it can be seen on Figs. 1-3, during each part of the simulation stages, the MSD are calculated from the initial positions of that part, i.e. a reset is operated after each change of thermodynamic ensemble. Consequently, the MSD increase or decrease that can be seen on these figures and that will be discussed in the following, corresponds to the maximum value reached at the end of each part.

\section{Results and discussion}

For several samples a drastic increase of Pb MSD was observed at some stages (see Fig. 1). This sudden increase of mobility is accompanied with an increase of the temperature indicating an exothermic transformation. Such transformations have been already seen in the case of the devitrification processes from amorphous to crystalline structures [4]. It is interpreted as a reorganization of the lead sublattice leading to a new structure.

As it was observed in the simulation of devitrification, the reorganization to a new crystalline structure is "prepared" by an important agitation of the fluorine ions. It is the case for the first transition $(\beta \rightarrow A)$ at 140 ps but not for the second one $(A \rightarrow B)$ at 560 ps. Once the cationic $\left(\mathrm{Pb}^{2+}\right)$ new sublattice $(A)$ is established the anions $\left(\mathrm{F}^{-}\right)$are quickly frozen (see Fig. 2 ). Checking the volume of the sample, we can see that the first transition $(\beta \rightarrow A)$ is accompanied with a slight decrease $(\sim-1 \%)$ of the volume and the second one $(\mathrm{A} \rightarrow \mathrm{B})$ with a slight increase $(\sim+3 \%)$. A-phase is denser than $\beta$ - and $B$-phases. It has to be compared with the experimental values of $10 \%$ mentioned by S. Hull and D.A. Keen [1] for the transition from $\alpha$ - to $\beta$-phases. Obviously these comparisons are only indicative for the direction of variations and not for the amplitude, since experimental and simulation conditions, pressure, temperature, relaxation of the system, are not strictly identical. The extreme mobility of fluorine ions in the $\beta$-phase before the first transition is consistent with the superionic conductivity of $\beta-\mathrm{PbF}_{2}$ since the conductivity is attributed to anion vacancies [2]. In Table 1 , in order to precise mobility values of fluorine ions, we have given the diffusion coefficient $\mathrm{D}$ calculated for each sample (one sample corresponds to one given temperature) in its initial pressure of 0 GPa ( $\beta$-phase). The obtained values are consistent with an Arrhenius law giving an activation energy of $0.52 \mathrm{eV}$. Values are comparable with those of Castiglione et al. [3].

In A-phase, fluorine ions are less mobile, as it could be the case in $\alpha-\mathrm{PbF}_{2}$. In $\mathrm{B}$-phase, the fluorine mobility remains weak, but the temperature is lower $(300 \mathrm{~K})$ than it was before the first transition $(>500 \mathrm{~K})$

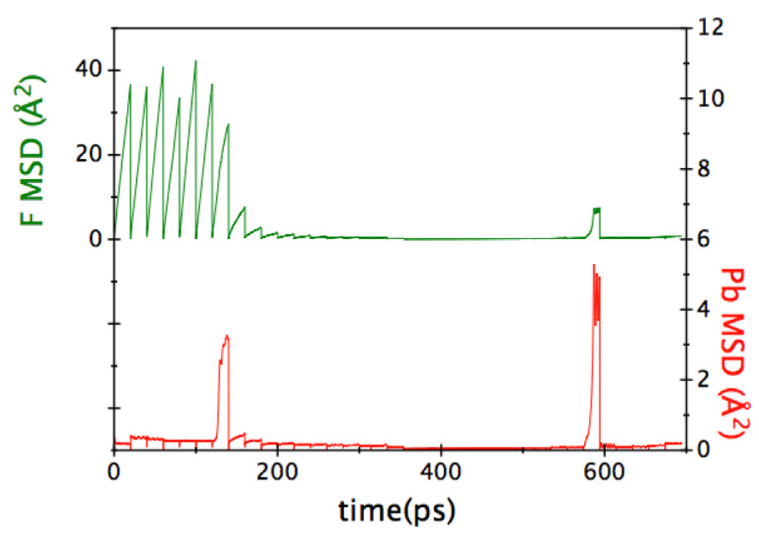

Fig. 2. Mean squared displacement for $\mathrm{Pb}^{2+}$ (bottom) and $\mathrm{F}^{-}$(top) ions. 


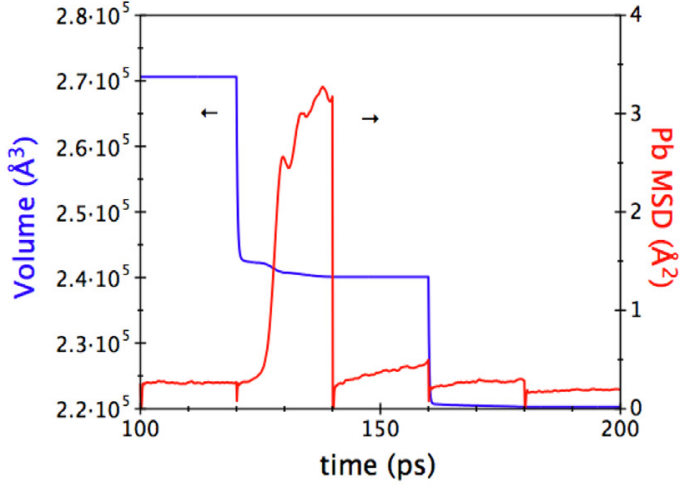

(a)

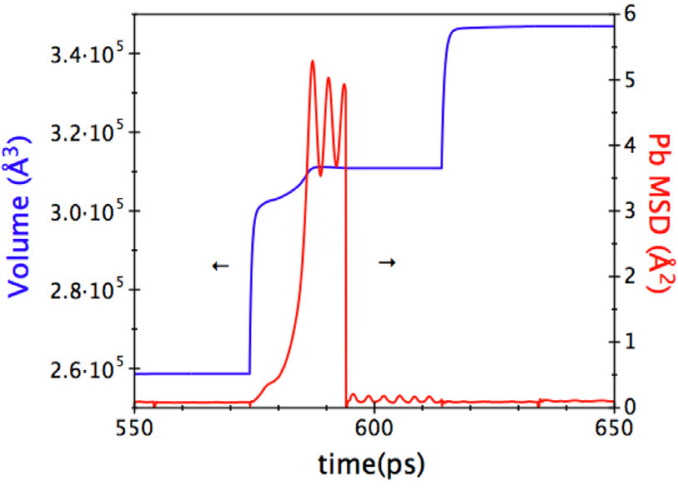

(b)

Fig. 3. Details of the transformations showing the volume changes accompanying the increase of mobility of $\mathrm{Pb}^{2+}$ ions for the phase transitions at $140 \mathrm{ps}$ (a) and 580 ps (b).

Table 1

Fluorine ion diffusion constant in function of the sample temperature at the first stages.

\begin{tabular}{clllllll}
\hline Temperature $(\mathrm{K})$ & 500 & 550 & 600 & 650 & 700 & 750 & 800 \\
$\begin{array}{c}\text { Diffusion coef. } \\
\left(10^{-9} \mathrm{~m}^{2} \mathrm{~s}^{-1}\right)\end{array}$ & 0.036 & 0.094 & 0.245 & 0.601 & 1.23 & 2.05 & 2.98 \\
\hline
\end{tabular}

In order to inspect and determine which are A and B structures, we have firstly plotted the $\mathrm{Pb}-\mathrm{F}$ RDF before and after the transformations (see Fig. 4).

Curves on Fig. 4(a) are very similar apart from the peak positions that are obviously different since the pressure and thus the volume are different. In consequence we can assert that the B-phase is a $\beta$ phase with a coordination distance of $2.39 \AA$ and a coordination number of 8 . The $\mathrm{Pb}-\mathrm{F}$ distance is shorter than the experimental one (2.57 $\AA$ ) due to change of volume. On Fig. 4(b), the A-phase is not as well organized as it appears: peaks are less sharp and consequently on CDF the plateau is absent. In that case, distances are not so well distinct that it is in the $\beta$-phase case. Just after the first peak, roughly at $3 \AA$, the coordination is higher than 8 . Whereas the cubic structure of $\beta-\mathrm{PbF}_{2}$ gives a single $\mathrm{Pb}-\mathrm{F}$ ionic distance, the orthorhombic one of $\alpha-\mathrm{PbF}_{2}$ phase gives nine different $\mathrm{Pb}-\mathrm{F}$ distances ranging from $2.41 \AA$ to $3.03 \AA$ [1]. The respective RDF are very similar to those we can calculate taking the crystallographic positions of ions in $\alpha-\mathrm{PbF}_{2}$ and $\beta-\mathrm{PbF}_{2}$ crystals (see Fig. 4(c)).

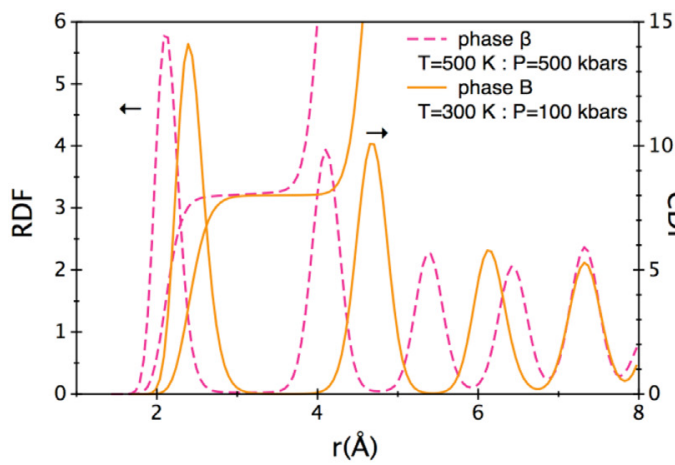

(a)

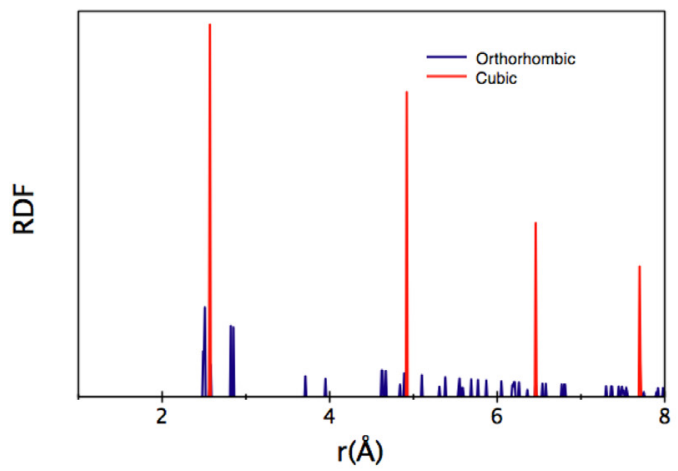

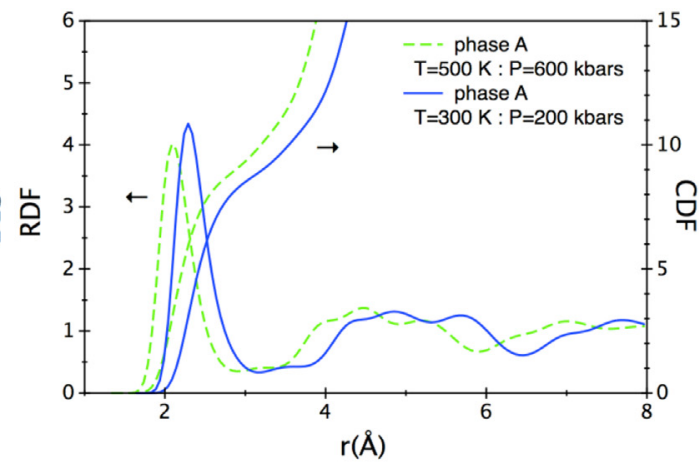

(b)

(c)

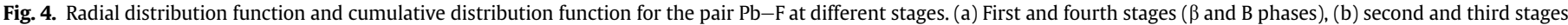

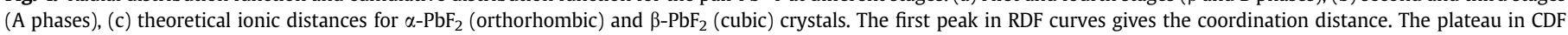
curves gives the coordination number. 


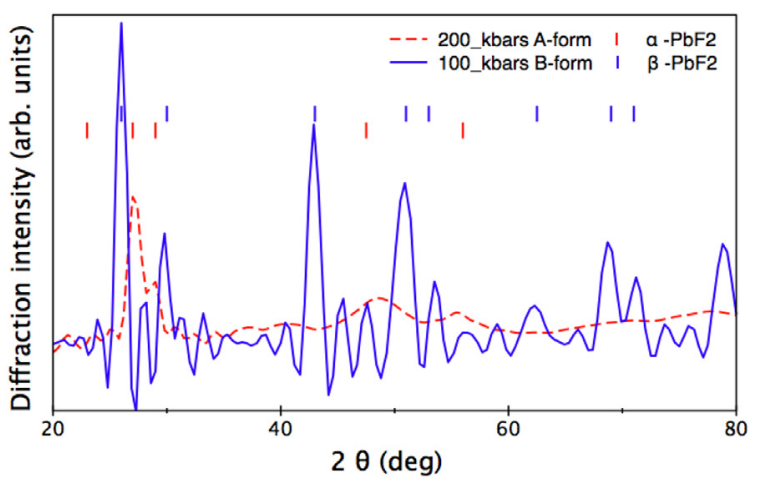

Fig. 5. XRD patterns of $A-(200$ kbars $)$ and $B-(100$ kbars $)$ forms identified to $\alpha-\mathrm{PbF}_{2}$ and $\beta-\mathrm{PbF}_{2}$. Spectra are plotted with a linear transformation of abscissa in order to take account of the volume contraction due to pressure effects.

In order to determine whether the A-phase corresponds to $\alpha$ $\mathrm{PbF}_{2}$ we have calculated X-ray diffraction spectrum using the simulated positions of the atoms and have compared it to the experimental ones (see Fig. 5). The diffracted intensity was modeled using the Debye formula $I(\theta)=\sum_{i, j} f_{i} f_{j}\left(\sin 2 \pi b r_{i j} / 2 \pi b r_{i j}\right)$, where $f_{i}$ and $f_{j}$ are the diffusion factors of atoms $i$ and $j, 2 \theta$ is the diffraction angle, $b=2 \sin \theta / \lambda$, and $r_{i j}$ is the distance between the two atoms $i$ and $j$. $\lambda$ was taken at $1.54 \AA$, corresponding to $K_{\alpha}(\mathrm{Cu})$.

The density of the simulated samples is higher than the experimental samples due to a higher pressure. For the B-phase we get the usual $\beta-\mathrm{PbF}_{2}$ diffraction spectrum with a slight shift for the peaks effectively due to the difference in density. The width of the peaks follows the Scherrer's formula with the crystal size [8]. The Aphase shows a spectrum with less distinct and intense peaks. This is the case for $\alpha-\mathrm{PbF}_{2}$. The 4 main structures are present with still a shift due to density discrepancy. We performed a linear correction to the abscissa $2 \theta$ to take into account the distance shortening due to the pressure. The corrected spectra are represented on Fig. 5.

Another possibility to check the structure is to determine the angular bonds distributions and to compare them to theoretical values of perfect crystals (see Fig. 6). The $\mathrm{F}-\mathrm{Pb}-\mathrm{F}$ angle gets three values in a cubic phase: $70^{\circ}, 110^{\circ}$ and $180^{\circ}$. That is clearly the case for $\beta-\mathrm{PbF}_{2}$. The same angle has more dispersed values in the ranges $65-80^{\circ}$ and $110-135^{\circ}$ in the orthorhombic phase. That is exactly the case for the A-form, i.e. $\alpha-\mathrm{PbF}_{2}$.

As a result of all our analysis there is no doubt that A-phase is $\alpha$ $\mathrm{PbF}_{2}$ and $\mathrm{B}$-phase is $\beta-\mathrm{PbF}_{2}$. Consequently we have induced phase transitions in our numerical samples.

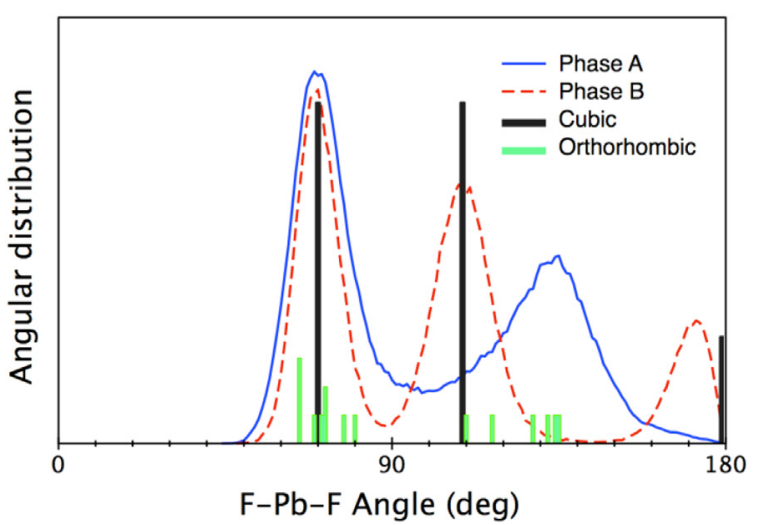

Fig. 6. $\mathrm{F}-\mathrm{Pb}-\mathrm{F}$ angular distribution functions for the $\mathrm{A}$ and $\mathrm{B}$-form compared to the perfect $\alpha-\mathrm{PbF}_{2}$ (orthorhombic) and $\beta-\mathrm{PbF}_{2}$ (cubic) crystals.
Table 2

Temperature and pressure for the phase transition $\beta \rightarrow \alpha$.

\begin{tabular}{llllllll}
\hline Temperature (K) & 500 & 550 & 600 & 650 & 700 & 750 & 800 \\
Pressure (GPa) & 60 & 60 & 40 & 30 & 30 & 30 & 30 \\
\hline
\end{tabular}

The temperature and pressure conditions where the phase transitions occur could give a $P-T$ phase diagram. However the applied pressure and temperature were incremented by large steps (10 GPa and $50 \mathrm{~K}$ ), giving us only a tendency and not accurate values (see Table 2).

In the range of experimented pressures (0-70 GPa), the $\beta \rightarrow \alpha$ transition is never achieved below $500 \mathrm{~K}$. Whatever the pressure, it is necessary to overstep $500 \mathrm{~K}$. On the other hand, the $\alpha \rightarrow \beta$ transition occurs always for $300 \mathrm{~K}$ at the pressure of $10 \mathrm{GPa}$. Though the simulated $\beta \rightarrow \alpha$ transition mimics the experimental one since it is induced by the pressure, the simulated $\alpha \rightarrow \beta$ transition is induced by the relaxation at lower temperature. But in experiments, this transition is accomplished by heating, and under ambient conditions $\mathrm{PbF}_{2}$ can exist in both forms [1]. The fact that in our simulated sample the $\alpha \rightarrow \beta$ transition comes from relaxation can be due to a less irreversible nature of the transformation in the numerical sample than in the real structure.

We address the question of what are the microscopic transformations leading to the phase transitions. A first answer has been provided by discussing the Fig. 2: we privileged a reorganization of the lead sublattice, which is followed by a freezing of the fluorine ions. To inspect more precisely the ionic displacements, we have recorded the ions positions every $0.1 \mathrm{ps}$ and averaged 10 successive positions in order to get an averaged positions every ps. For a given lead ion, we plot a snapshot of its 1 st $\left(\mathrm{F}^{-}\right)$and $2 \mathrm{nd}\left(\mathrm{Pb}^{2+}\right)$ nextneighbors for $20 \mathrm{ps}$, before, during and after the transformation. Snapshots are represented on Fig. 7. The displacements of $\mathrm{Pb}^{2+}$ are limited and the change in crystalline structure is hidden by the displacements due to the lattice dilatation or contraction due to pressure and/or temperature changes. There is no hop of $\mathrm{Pb}^{2+}$ from one crystallographic site to another, suggesting that the transformation from one crystalline sublattice to the other is a continuous deformation process.

Fluorine ions, for their part, are continuously moving, hoping quickly from one crystallographic site to another during the transformations until they relatively freeze at the end. The important fluorine thermal mobility reaches a point where it relaxes the lead sublattice and allows a reorganization of the lead ions more compatible with the pressure and temperature constraints. This reorganization can be seen both as a slow displacements of $\mathrm{Pb}^{2+}$ ions or as a deformation process.

The fluorine mobility, as it can be seen on Fig. 2, is much more important for $\beta-\mathrm{PbF}_{2}$ than for $\alpha-\mathrm{PbF}_{2}$ : compare the F- MSD before and after the $\beta \rightarrow \alpha$ transition, in line with the superionic character of $\beta-\mathrm{PbF}_{2}$. Castiglione et al. [3] have explained, after computer simulations using polarizable ionic potentials, that the ionic motions are unlikely for $\alpha$ - and $\beta-\mathrm{PbF}_{2}$, and particularly via a hopping process for $\alpha$-phase. Our less refined simulation gives also a quantitative difference for the ionic mobility in each phase. Moreover it has been successful in reproducing phase transitions.

\section{Conclusion}

Phase transitions between two crystalline structures in lead fluoride have been simulated using classical molecular dynamics. All the techniques of analysis applied to our numerical samples have demonstrated a pressure-induced transition from a cubic to an orthorhombic phase. Even if temperature and pressure conditions are slightly different from the experimental ones, it is 


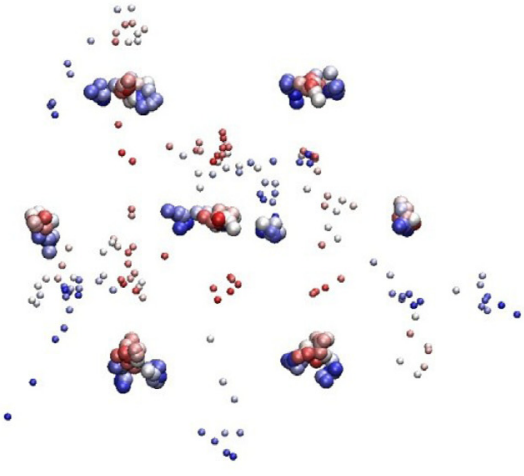

(a)

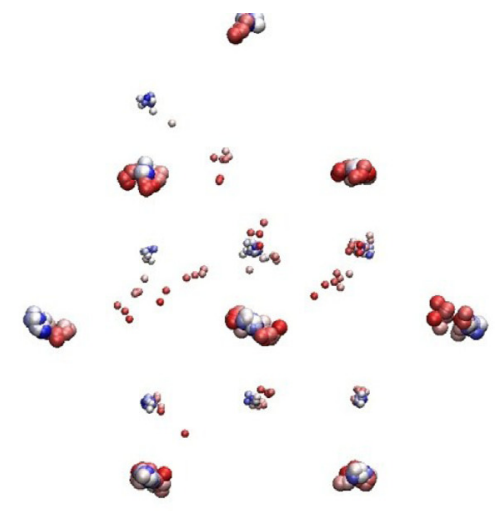

(b)

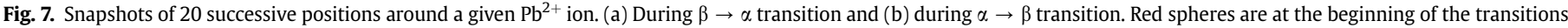

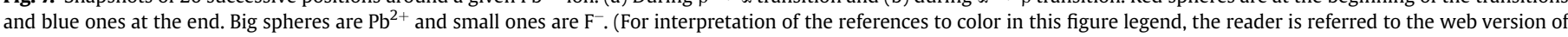
this article.)

demonstrated that $\beta$ (cubic) $\rightarrow \alpha$ (orthorhombic) transition occurs with an increasing pressure. The process is characterized in terms of ionic mobility leading to a reorganization of crystalline networks.

\section{References}

[1] S. Hull, D.A. Keen, Phys. Rev. B 58 (1998) 14837.

[2] See e.g. N.I. Sorokin, P.P. Fedorov, B.P. Sobolev Inorg. Mater. 33 (1997) 5

[3] M.J. Castiglione, M. Wilson, P.A. Madden, C.P. Grey, J. Phys.: Condens. Matter 13 (2001) 51. M.J. Castiglione, P.A. Madden, J. Phys.: Condens. Matter 13 (2001) 9963.
[4] M.A.P. Silva, A. Monteil, Y. Messaddeq, S.J.L. Ribeiro, J. Chem. Phys. 117 (2002) 5366.

[5] A.B. Walker, M. Dixon, M.J. Gillan, J. Phys. C 15 (1982) 4061.

[6] R. Martonak, A. Laio, M. Parrinello, Phys. Rev. Lett. 90 (2003) 075503. R. Martonak, D. Donadio, A.R. Oganov, M. Parrinello, Nat. Mater. 5 (2006) 623.

[7] I.T. Todorov, W. Smith, K. Trachenko, M.T. Dove, J. Mater. Chem. 16 (2006) 19111918.

[8] G. Dantelle, M. Mortier, A. Monteil, S. Chaussedent, M.A.P. Silva, J. Chem. Phys. 127 (2007) 094509. 